\title{
Phenotypic and genotypic detection of extended spectrum $\beta$-lactamases among Escherichia coli and Klebsiella pneumoniae isolates from type 2 diabetic patients with urinary tract infections
}

\author{
Souad Youssouf Kani Elmi ${ }^{1}$, Medhat Saber Ashour ${ }^{2}$, Fathy Zakaria Alsewy ${ }^{3}$, \\ Nashwa Fawzy Abd El Moez Azzam²
}

1. Master Degree in Public Health, High Institute of Public Health, Alexandria University. Egypt. Bachelor's Degree in Medical Laboratories, Faculty of Medicine and Health Sciences, Sana'a University. Yemen.

2. High Institute of Public Health, Alexandria University. Egypt.

3. Department of Diabetes and Metabolism, Faculty of Medicine, Alexandria University.Egypt.

\begin{abstract}
Background: T2DM patients are more likely to have UTIs caused by resistant organisms such as ESBLs producing bacteria. Challenging reliable identification and prompt characterization of in-vitro susceptibilities of these bacteria are the first steps of deciding the appropriate antimicrobial therapy for UTIs caused by them.

Objectives: To isolate and identify E. coli and K. pneumoniae from urine of T2DM patients with UTIs, to determine antibiotic resistance pattern among isolates, and to identify ESBLs production phenotypically and genotypically.

Material and method: All samples were cultured on Cystine-Lactose-Electrolyte-Deficient Agar medium (CLED) by using calibrated loop. Growth of 100 colonies or more, i.e. 105 colony forming units (CFU)/mL urine was considered as significant bacteriuria. Isolation and identification were done according to standard method. All isolates were tested for antibiotic susceptibility testing by the disc diffusion method according to CLSI guidelines. Phenotypic detection of ESBLs was done by double-disk synergy test. Genotypic detection of blaTEM, blaSHV and blaCTX-M genes by using PCR.

Results: Results of this study showed that E. coli and K. pneumoniae were the dominant bacterial isolates, they constituted 103 (91.2\%) out of 113 urine isolates. E. coli (58. 4\%) K. pneumoniae (32.7\%), Enterococcus spp. (4.4\%), Proteus spp. (2.7\%) and Pseudomonas spp. (1.8\%). About 25 (24.3\%) out of 103 E. coli and K. pneumoniae isolates were ESBLs positive by DDST, and $22(88.0 \%)$ out of them had ESBLs encoding genes by conventional PCR. The most common gene detected was blaTEM (59.1\%), followed by blaSHV (27.3\%). CTX-M had not been detected in any of testes isolates.

Conclusion: blaTEM and blaSHV genes were detected in 22 out of 25 ESBLs producing E. coli and K. pneumoniae isolates phenotypically detected by DDST. blaTEM was found to be the predominant gene (59.1\%), while blaCTX-Mene was not detected in any of tested isolates.

Keywords: Extended Spectrum $\beta$-Lactamases, Type 2 diabetes mellitus, Urinary tract infections, Phenotypic and genotypic methods.

DOI: https://dx.doi.org/10.4314/ahs.v21i2.3

Cite as: Elmi SYK, Ashour MS, Alsewy FZ, Azzam NFA-M. Phenotypic and genotypic detection of extended spectrum $\beta$-lactamases among Escherichia coli and Klebsiella pneumoniae isolates from type 2 diabetic patients with urinary tract infections. Afri Health Sci. 2021;21(2). 497-504. bttps://dx.doi.org/10.4314/abs.v21i2.3
\end{abstract}

\section{Introduction}

Type 2 diabetes mellitus (T2 DM) is a metabolic disorder characterized by variable degrees of insulin resistance, impaired insulin secretion, and increased glucose production ${ }^{1}$. Patients with T2DM are at increased risk

\section{Corresponding author:}

Nashwa Fawzy Abd El Moez Azzam,

Associate professor of Microbiology,

High Institute of Public Health,

Alexandria University. Egypt.

Email: nashwaazam@yahoo.com for urinary tract infections (UTIs) due to diabetic neuropathy that causes dysfunctional voiding and urinary retention, thus facilitating bacterial growth. High glucose content of urine provide nutrition for urinary microbes $^{2}$. Patients with diabetes are more likely to have resistant organisms causing UTIs, including extended spectrum $\beta$-lactamases(ESBLs), carbapenem-resistant Enterobacteriaceae $e^{3-5}$. Based on the synergy between a third-generation cephalosporin and clavulanate, several phenotypic detection methods of ESBLs had been designed; such as double-disk synergy test (DDST) ${ }^{6}$. Alternative strategies to replace traditional phenotyp- 
ic methods had been proposed; the most widely used techniques are conventional polymerase chain reaction (PCR) and gene sequencing ${ }^{7,8}$.

\section{Material and methods}

\section{Study area}

The study was carried out over a period of four months from May 2019 to August 2019. It enrolled 113 type 2 diabetic patients suffering from UTIs attending the outpatient clinics of the Department of Diabetes and Metabolism or Department of Urology at Alexandria Main University Hospital. Alexandria city. Egypt.

\section{Laboratory investigation \\ Sampling}

Clean catch midstream urine samples were aseptically collected from 113 type 2 diabetic patients with UTIs. Media-Test Combi10 reagent dipsticks were used for chemical urine analysis. They contain up to 10 different chemical pads or reagents (blood, urobilinogen, bilirubin, protein, nitrate, ketones, glucose, $\mathrm{pH}$, specific gravity and leukocytes).

Semi-quantitative urine culture on CLED agar and biochemical identification of bacterial isolates were done9. All E. coli and K. pneumoniae isolates were tested for their antibiotic susceptibility using the Kirby Bauer disc diffusion method. Antibiotics used were Ampiillin, Cefaclor, Cefotaxime, Ceftazidime, Cefepime, Amoxicillin-clavulanic acid, Aztreonam, Imipenem, Amikacin, Norfloxacin and Nitrofurantoin. Inhibition zones were measured, and then susceptibility was recorded as susceptible (S), intermediate (I) and resistant (R) according to CLSI breakpoints.

\section{Double-disc synergy test}

A lawn culture of each E. coli and K. pneumoniae isolates was made on MHA plate, as recommended by CLSI. A disc of amoxicillin-clavulanic acid $(20 / 10 \mu \mathrm{g})$ was placed in the center of the plate at $20 \mathrm{~mm}$ distance to

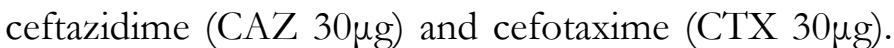
ESBLs production was detected by the appearance of keyhole effect due to the enhanced activity of ceftazidime and cefotaxime with clavulanic acid ${ }^{10}$.

Genotypic method for detection of ESBLs Producing E. coli and $K$. pneumoniae by conventional Polymerase Chain Reaction (PCR).

Genomic DNA was extracted using a DNA easy tissue kit (Qiagen, Germany) according to the instructions of the manufacturer.

\section{DNA Amplification protocol}

Initial denaturation $\left(94^{\circ} \mathrm{C}\right.$ for $\left.5 \mathrm{~min}\right)$; 30 cycles of denaturation $\left(94^{\circ} \mathrm{C}\right.$ for $\left.30 \mathrm{~s}\right)$, annealing $\left(58^{\circ} \mathrm{C}\right.$ for $\left.1 \mathrm{~min}\right)$ and polymerization $\left(72^{\circ} \mathrm{C}\right.$ for $\left.1 \mathrm{~min}\right)$; and an additional polymerization step $\left(72^{\circ} \mathrm{C} \text { for } 7 \mathrm{~min}\right)^{11}$.

\section{DNA detection}

The amplification products were analysed by agarose gel electrophoresis and ethidium bromide staining ${ }^{12}$. The gel running plate was placed in its special gel casting plate supplied with electrophoresis apparatus (MupidExu submarine electrophoresis system, Advance, Japan)

\section{Results}

The majority of T2DM patients with UTIs were females $(69 \%)$. Most of female patients, $(47.4 \%)$ were of age group 55-65 years. $97.3 \%$ of cultured clean catch midstream urine samples yield significant bacteriuria, where $70 \%$ were cultured from female patients, and 30\% from male patients. Glucose concentration in urine ws found to significantly affect the colony count of urine cultures, where $(80.9 \%)$ urine samples with colony count $\geq 105 \mathrm{CFU} / \mathrm{ml}$ were cultured from T2DM patients with +3 glucosuria level $(\geq 500 \mathrm{mg} \backslash \mathrm{dl})$. E. coli and K. pneumoniae isolates were the most prevalent uropathogens, they had been isolated from $103(91.2 \%)$ out of 113 UTIs in T2DM. table (1) 
able 1: Common etiologic agents of UTIs in T2DM patients.

\begin{tabular}{|c|c||}
\hline Etiologic agents & No. (\%) \\
\hline \hline E. coli & $66(58.4 \%)$ \\
\hline K. pneumoniae & $37(32.7 \%)$ \\
\hline Enterococci & $5(4.4 \%)$ \\
\hline Pseudomonas aeruginosa & $2(1.8 \%)$ \\
\hline Proteus spp & $3(2.7 \%)$ \\
\hline Total & $\mathbf{1 1 3}(\mathbf{1 0 0 \% )}$ \\
\hline
\end{tabular}

About $24.3 \%$ of UTIs caused by E. coli and K. pneumoniae isolates were due to ESBLs-producing strains. The highest percentage of ESBL-UTIs (68\%) were found in age groups of $55-65$ years, and $80 \%$ of them were females. Duration of diabetes and poor glycemic control were not found to significantly increase the risk of ESBLs, however use of urinary catheter and prior use of antibiotics were found to be associated with ESBLs positivity. ESBLs production appeared as a significant risk factor for acute kidney injury.

Table 2: Distribution of E. coli and K. pneumoniae isolates according to ESBLs production.

\begin{tabular}{|c|c|c|c|}
\hline \multirow[t]{3}{*}{ ESBLs production } & \multicolumn{2}{|c|}{ Isolates } & \multirow[t]{2}{*}{ Total } \\
\hline & E. coli & K. pneumoniae & \\
\hline & No. (\%) & No. (\%) & No. (\%) \\
\hline ESBL & $17(25.8 \%)$ & $8(21.6 \%)$ & $25(24.3 \%)$ \\
\hline Non ESBL & $49(74.2 \%)$ & $29(78.4 \%)$ & $78(75.7 \%)$ \\
\hline Total & $66(100 \%)$ & $37(100 \%)$ & $103(100 \%)$ \\
\hline
\end{tabular}




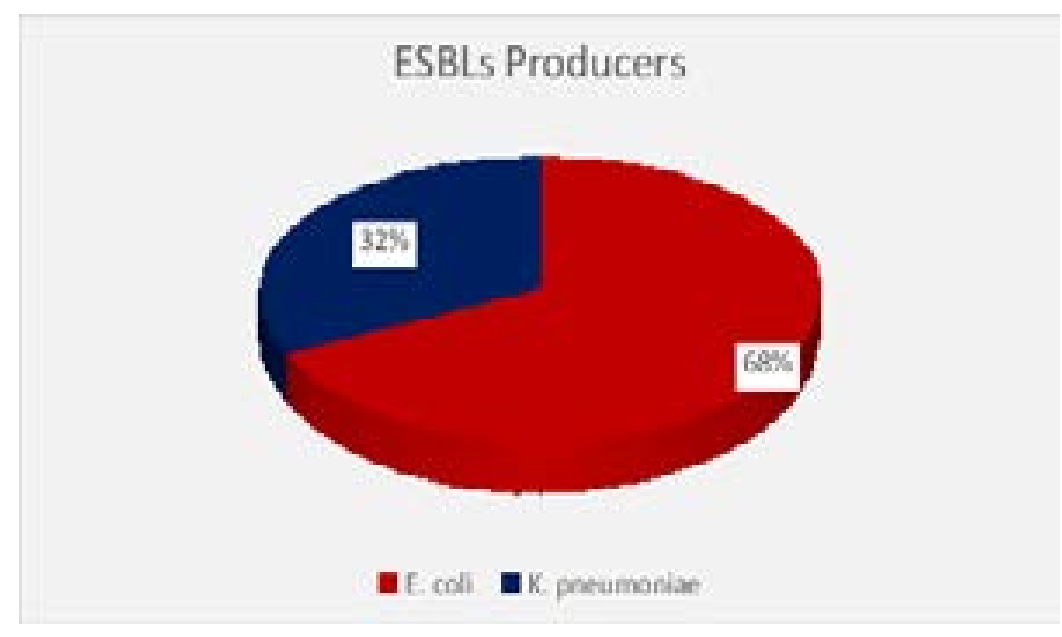

Figure 1: Distribution of ESBLs- producing urine isolates detected by DDST.

ESBLs producing isolates showed higher resistance crease was significant with aztreonam, all cephalosporrates to antibiotics than non ESBLs producing, this inin, and amoxicillin clavulanate. Figure (2)

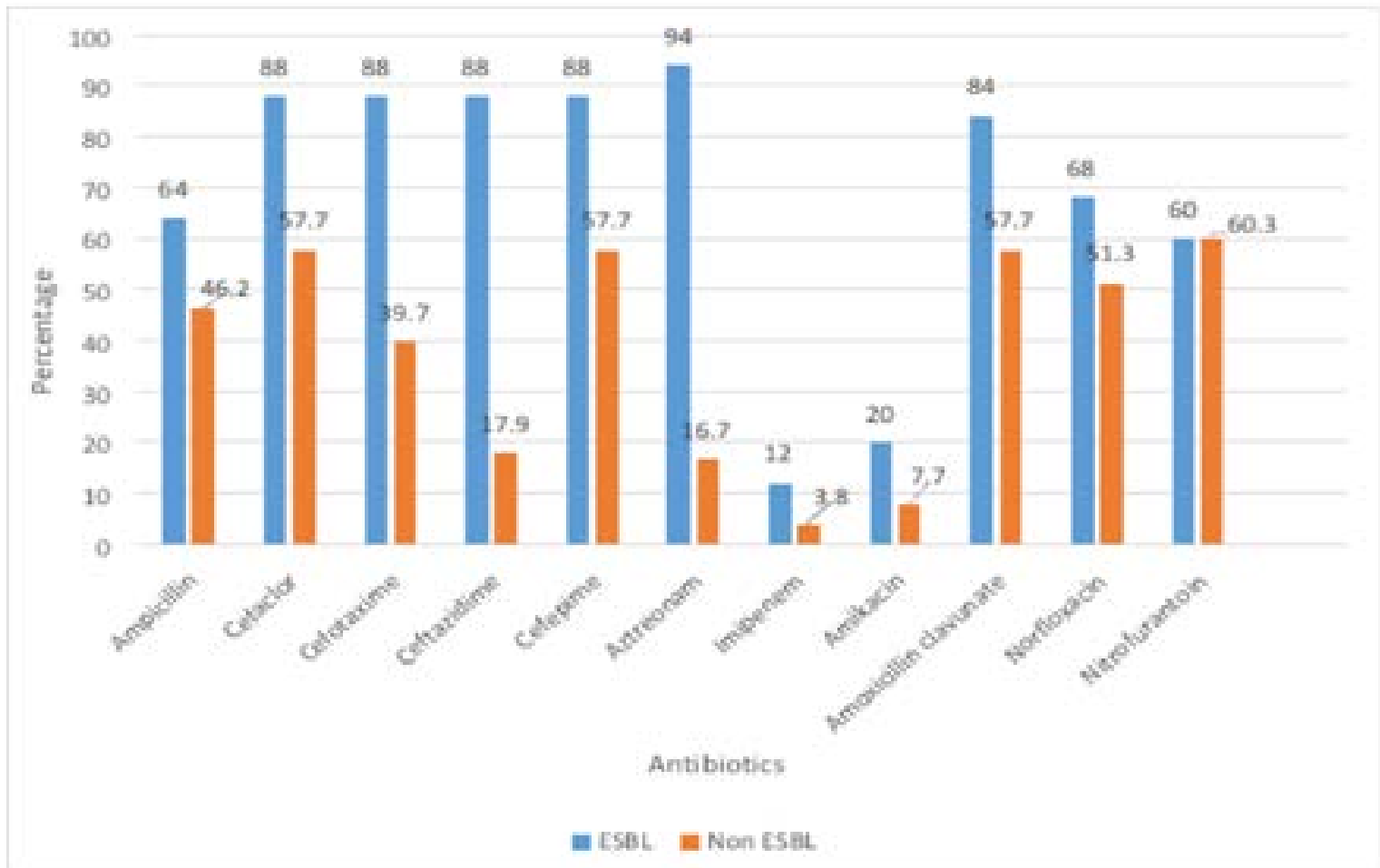

Figure 2: Antibiotics resistance patterns of ESBL and non ESBL isolates

ESBLs genes were detected in only 22 out of 25 ESBLs producing isolates detected by DDST phenotypic method.

Three isolates (one E. coli and two K. pneumoniae isolates) were not confirmed by PCR to have ESBLs encoding genes.
The predominant gene detected was blaTEM, as it was detected in $13(59.1 \%)$ out of 22 confirmed ESBLs producing isolates. blaSHV was detected in $6(27.3 \%)$ out of 22 confirmed ESBLs producing isolates. Three isolates harbored both genes (13.6\%). blaCTX was not detected in any of ESBLs producing isolates. Table (3) 
Table 3: Prevalence of ESBLs genes among ESBLs producing isolates.

\begin{tabular}{||c|c|c|c||}
\hline \multirow{2}{*}{ ESBL genes } & \multicolumn{2}{|c|}{ Isolates } & \multirow{2}{*}{ Total } \\
\cline { 2 - 4 } & E. coli (n=16) & K. pneumoniae (n=6) & \\
& & & \\
& & & \\
& & & No (\%) \\
\hline \hline TEM & $11(68.8 \%)$ & $2(33.3 \%)$ & $13(59.1 \%)$ \\
\hline SHV & $3(18.7 \%)$ & $3(50 \%)$ & $6(27.3 \%)$ \\
\hline TEM+SHV & $2(12.5 \%)$ & $1(16.7 \%)$ & $3(13.6 \%)$ \\
\hline
\end{tabular}

\section{Discussion}

T2DM is a reported risk factor for frequent and severe UTIs due to depressed immunity, altered phagocytic adhesion and abnormal physiological process. Wide use of broad- spectrum antibiotics to treat these infections facilitates growth of antibiotic resistant urinary pathogens such as ESBLs-producing bacteria, which imposes substantial burden on medical cost due to increased risk of worse outcomes of UTIs caused by these bacteria. Post-menopausal women found to have much higher risk of T2DM-UTIs because drop in estrogen level is associated with insulin resistance and faulty regulation of metabolic homeostasis ${ }^{13}$. Majority of T2DM patients with UTIs in the present study $(69 \%)$ were females, and the highest percentage of them $(47.4 \%)$ were in post-menopausal period (55-65 years). This agreed with previous studies done by Zubair et al., ${ }^{14}$ and Patra et al. from India, ${ }^{15}$ On the contrary, Mogaka et al. (2018) from Kenya ${ }^{16}$, and Sibi et al. ${ }^{17}$ from India found that diabetic male were affected with UTIs more than women. Most of clean catch urine cultures from T2DM patients had significant bacteriuria (97.3\%), this was in accordance with a study by Longdoh et al. ${ }^{18}$ from Cameroon $(81.6 \%)$. A high frequency of significant bacteriuria was found among female diabetic patients $(70 \%)$, this was in agreement with study obtained by Patra et al. ${ }^{15}$ from
India. There was a significant association between glucose concentration in urine and culture colony count, where $80.9 \%$ of urine samples with colony count $\geq 105$ $\mathrm{CFU} / \mathrm{ml}$ were cultured from T2DM patients with +3 glucosuria level ( $\geq 500 \mathrm{mg} \backslash \mathrm{dl}$ ). This study was in accordance with findings by Selim et al. (2019)19 from Egypt who detected bacterial growth in $100 \%$ of urine samples from patients with +4 glucosuria level. Mono microbial etiology of UTIs was found to be more common in this study, where E.coli was the most prevalent organism (58.4\%); similar findings had been reported by Zubair et al. (2019) ${ }^{14}$ from Pakistan and Rahim et al. ${ }^{20}$ from Bangladesh. Out of 103 UTIs caused by E.coli and $K$. pneumoniae, $25(24.3 \%)$ were due to ESBLs producing isolates. These findings were comparable to findings reported by Onanuga et al. (2019) from Nigeria, ${ }^{21}$ Abayneh et al. ${ }^{22}$ from Ethiopia. In the present study more than two thirds $(68 \%)$ of ESBLs producing isolates were E. coli; and similar finding been reported in several studies ${ }^{22-24}$. More than one fourth of E. coli $(25.8 \%)$ and $21.6 \%$ of $K$. pneumoniae isolates were found to be ESBL- producers. Significant association between ESBLs positivity and advanced age, use of urinary catheters and misuse of antibiotics. This observation was parallel to other studies ${ }^{25-27}$. In the present study, ESBLs production appeared as a significant risk factor for 
acute kidney injury, where all patients with history of AKI had ESBL-UTIs (100\%). Same finding were reported by Rahim et al. (2018) from Bangladesh ${ }^{20}$ and Ramadas et al. (2014) from New York ${ }^{28}$. The results of antimicrobial susceptibility revealed that E. coli showed high resistance percentages to ampicillin (78.8\%), followed by cefaclor (63.6\%), norfloxacin (57.6\%) and nitrofurantoin (54.6\%). Zubair et al. (2019) showed similar resistance percentages to cefaclor, norfloxacin and nitrofurantoin $(50 \%, 62.7 \% \text { and } 56.8 \% \text {, respectively })^{14}$.

$K$. pneumoniae isolates showed high resistance percentages to nitrofurantoin $(70.3 \%)$, cefaclor $(67.6 \%)$ and norfloxacin $(51.4 \%)$. This high level of resistance to norfloxacin and nitrofurantoin could be explained by absence of strict antibiotics use policy in the community, added to the exponential increase in prescription of these antibiotics for empiric treatment of hospitalized patients with positive urine cultures. The antimicrobial profile of ESBLs producing isolates showed significantly higher resistanceo aztreonam, all cephalosporin, and amoxicillin clavulanate compared to non-ESBLs producing isolates.

bla'TEM was found to be the predominant gene (59.1\%) in ESBL isolates, this finding was consistent with those found by Fattouh et al. ${ }^{29}$ from Egypt. blaCTX-M gene was not detected in any of tested isolates. Most of $\mathrm{E}$. coli isolates harbored blaTEM gene (68.8\%), while half of tested $\mathrm{K}$. pneumoniae isolates had blaSHV gene. Plasmid-encoded ESBLs-producing bacteria can show co-resistance to quinolones, aminoglycosides and sulfonamide. In the present study $69.8 \%$ and $38.5 \%$ of isolates harboring blaTEM gene were found to be resistant to norfloxacin and amikacin, respectively. Isolates harboring blaSHV showed lower resistant rates to norfloxacin and nitrofurantoin (33.3\%) and full sensitivity to amikacin. In the present study, the observed high level of resistance to imipenem (46.2\%) among blaTEM harboring isolates is worrisome, since carbapenems are the treatment of choice for these isolates. In the current study, three isolates that were phenotypically positive by DDST method lacked TEM, SHV, and/ or CTX-M genes. This may be explained by that these three isolates may carry other ESBLs encoding genes, which coud not be detected by the used primers, or it could be chromosomally mediated AmpC $\beta$-lactamases. This finding was parallel to that reported by Bajpai et al. ${ }^{30}$ from India. However, Sowmiya et al. (2012) from India showed a 100\% correlation between phenotypic and genotypic methods of ESBLs detection ${ }^{31}$.

\section{Authors' contribution}

Supervision of the implementation and findings of this work, contribution in interpretation of the results. ${ }^{2,3}$

Supervision of the microbiological laboratory procedure, contribution in data analysis, interpretation of results and writing of manuscript. ${ }^{4}$

Implementation of the microbiological laboratory procedure, contribution in analysis of the results, and writing manuscript. ${ }^{1}$

All authors discussed the results and contributed to the final manuscript.

\section{References}

1. Organization, W. H. (2017). Definition and Diagnosis of Diabetes Mellitus and Intermediate Hyperglycemia. . Retrieved from Geneva, Switzerland:

2. Chen, SL., Jackson, SL., Boyko, EJ. (2009). Diabetes mellitus and urinary tract infection: epidemiology, pathogenesis and proposed studies in animal models. Journal of Urology, 182(6 Suppl): S51-S6.

3. Shah, AA., Hasan, F., Ahmed, S., \& Hameed, A. (2004). Characteristics, epidemiology and clinical importance of emerging strains of gram negative bacilli producing extended-spectrum beta-lactamases. Research in Microbiology, 155: 409-21.

4. Wayne, PA . (2009). M100-S19 Performance Standards for Antimicrobial Susceptibility Testing. 19th ed. Clinical and Laboratory Standards Institute (CLSI), p 250 .

5. Lee, J., Pai, H., Kim, YK., Kim, NH., Eun, BW., \& Kang ,HJ. (2007). Control of extended spectrum beta-lactamase producing Escherichia coli and Klebsiella pneumoniae in a children's hospital by changing antimicrobial agent usage policy. Journal of Antimicrobial Chemotherapy, 60: 629-37.

6. Drieux, L., Brossier, F., Sougakoff1, W., \& Jarlier, V. (2008). Phenotypic detection of extended-spectrum $\beta$-lactamase production in Enterobacteriaceae: review and bench guide. Clinical of Microbiology and Infection, 14(Suppl.1): 90-103.

7. Cole , JM., Schuetz, AN., Hill ,CE., \& Nolte, FS. (2009). Development and evaluation of a real-time PCR assay for detection of Klebsiella pneumoniae carbapenemase genes. Journal of Clinical Microbiology, 47: 322-6. 8. Jones, CH., Ruzin, A., Tuckman, M., Visalli, MA., Petersen, PJ., \& Bradford, PA. (2009). Pyro sequencing using the single-nucleotide polymorphism protocol for rapid determination of TEM- and SHV type extended-spectrum $\beta$-lactamases in clinical isolates and identification of the novel $\beta$-lactamase genes blaSHV-48, blaSHV-105, and blaTEM-155. Antimicrobial Agents Chemotherapy, 53: 977-86. 
9. Bauer, A., Kirby, W., Sherris, J. C., \& Turck, M. (1966). Antibiotic susceptibility testing by a standardized single disk method. American Journal of Clinical pathology, 45(4 ts), 493-496.

10. Wayne, PA. (2018). M100 E28 Performance standards for antimicrobial susceptibility testing. 28th ed. Clinical and Laboratory Standards Institute (CLSI), p 250.

11. Amine, A. E. K. (2013). Extended spectrum beta-lactamase producing bacteria in waste water alexandria, Egypt. International Journal of Bioscience, Biochemistry and Bioinformatics, 3(6), 605-9.

12. Lee, P. Y., Costumbrado, J., Hsu, C.-Y., \& Kim, Y. H. (2012). Agarose gel electrophoresis for the separation of DNA fragments. JoVE (Journal of Visualized Experiments.(62), e3923.

13. Nitzan, O., Elias, M., Chazan, B., \& Saliba, W. (2015). Urinary tract infections in patients with type 2 diabetes mellitus: review of prevalence, diagnosis, and management. Diabetes, metabolic syndrome and obesity: targets and therapy

14. Zubair, K. U., Shah, A. H., Fawwad, A., Sabir, R., \& Butt, A. (2019). Frequency of urinary tract infection and antibiotic sensitivity of uropathogens in patients with diabetes. Pakistan Journal of Medical Sciences, 35(6).

15. Patra, E. P., Karna, S., Meher, D., \& Mishra, S. (2019). Bacterial causes of community-acquired and nosocomial urinary tract infection in type 2 diabetes: A comparative approach. Journal of Diabetology, 10(3), 102. 16. Mogaka, M. V. (2018). Determination of Urinary Tract Infections and Antibiotic Sensitivity among Non-Insulin Dependent Diabetes Mellitus Patients Visiting Kisii Teaching And Referral Hospital, Kenya. Kenyatta University.

17. Sibi, G., Devi, A. P., Fouzia, K., \& Patil, B. R. (2011). Prevalence, microbiologic profile of urinary tract infection and its treatment with trimethoprim in diabetic patients. Research Journal of Microbiology, 6(6), 543-551.

18. Longdoh, N. A. (2013). Uropathogens from diabetic patients with asymptomatic bacteriuria and urinary tract infections. West London Medical Journal, 5(1), 7-14. 19. Selim, S., Abdel Aziz, M., El-Alfay, S., \& Zakaria, H. (2019). Incidence and Antibiotics Resistance of Staphylococci and Escherichia coli Isolated from Diabetic Urinary Tract Infection Patients in Egypt. Journal Pure Appl Microbiol, 13 (3), 1697-1702.

20. Rahim, M. A., Mitra, P., Haque, A., Zaman, S., Samad, T., Haque, W. M. M. U., . . Sarker, S. (2018). Urinary Tract Infection due to Extended-Spectrum Beta-Lactamase Producing Organisms is a Risk Factor for Acute
Kidney Injury among Patients with Type 2 Diabetes Mellitus. Journal of Medicine, 19(1), 40-43.

21. Onanuga, A., Mahindroo, J., Singh, S., \& Taneja, N. (2019). Phenotypic and molecular characterization of antimicrobial resistant Escherichia coli from urinary tract infections in Port-Harcourt, Nigeria. Pan African Medical Journal, 34(144)

22. Abayneh, M., Tesfaw, G., \& Abdissa, A. (2018). Isolation of Extended-Spectrum $\beta$-lactamase-(ESBL-) Producing Escherichia coli and Klebsiella pneumoniae from Patients with Community-Onset Urinary Tract Infections in Jimma University Specialized Hospital, Southwest Ethiopia. Canadian Journal of Infectious Diseases and Medical Microbiology, 2018.

23. Eltai, N. O., Al Thani, A., A. , Al-Ansari, K., Deshmukh, A. S., Wehedy, E., Al-Hadidi, S. H., \& Yassine, H. M. (2018). Molecular characterization of extended spectrum $\beta$-lactamases enterobacteriaceae causing lower urinary tract infection among pediatric population. Antimicrobial Resistance and Infection Control, 7: 90, 2-9. doi: doi: 10.1186/s13756-018-0381-6

24. Shakya, P., Shrestha, D., Maharjan, E., Sharma, V. K., \& Paudyal, R. (2017). ESBL production among E. coli and Klebsiella spp. causing urinary tract infection: a hospital based study. The Open Microbiology Journal, 11, 23-30.

25. Iqbal, S., Rahim, M. A., Samad, T., Ananna, M. A., Mitra, P., \& Chowdhury, T. A. (2015). Extended-Spectrum Beta-Lactamase Producing Escherichia coli and Klebsiella pneumoniae are Emerging as Major Pathogens Responsible for Urinary Tract Infection. Bangladesh Critical Care Journal, 3(2), 49-52.

26. Saedii F, A., Hakeem, M., \& Abdelraheem, A. (2017). ESBL-Producing E. coli and Klebsiella among Patients Treated at Minia University Hospitals. Journal of Infectious Diseases \& Preventive Medicine, 5(2), 1000156.

27. Goyal, D., Dean, N., Neill, S., Jones, P., \& Dascomb, K. (2019). Risk Factors for Community-Acquired Extended-Spectrum Beta-Lactamase-Producing Enterobacteriaceae Infections-A Retrospective Study of Symptomatic Urinary Tract Infections. Indian Journal of Medical Research, 6(2), 1-6.Goyal (2019)

28. Ramadas P, Rajendran PP, Krishnan P, Alex A, Siskind E, Kadiyala A, et al. Extended-Spectrum-Beta-Lactamase Producing Bacteria Related Urinary Tract Infection in Renal Transplant Recipients and Effect on Allograft Function. PLoS One. 2014;9(3):e91289.

29. Fattouh M., El-din A. N. Emergence of carbapenem-resistant acinetobacter baumannii in the intensive care unit in Sohag University Hospital, Egypt. Interna- 
tional Journal of Current Microbiology and Applied Sciences. 2014;3(4):732-744.

30. Bajpai T, Pandey M, Varma M, Bhatambare GS. Prevalence of TEM, SHV and CTX-M beta-lactamase genes in the urinary isolates of a tertiary care hospital. Avicenna J Med. 2017;7:12-16. doi: 10.4103/22310770.197508.
31. Sowmiya M, Malathi J, Madhavan HN. Screening of ocular Enterobacteriaceae isolates for presence of chromosomal blaNDM-1 and ESBL genes: A 2-year study at a tertiary eye care center. Invest Ophthalmol V is Sci. 2012;53:5251-7 